\title{
Relación del estado nutricional de las madres y la alteración de los factores de riesgo cardiovascular de sus hijos
}

\author{
Karina Rozas ${ }^{1 a}$, Patricio Huerta ${ }^{1 b}$, Josefa Bahamonde ${ }^{1 a}$, Constanza Atenas $^{1 a}$, Tatiana Urra ${ }^{1 a}$, María Virginia Araya ${ }^{2 c}$ \\ 1.-Universidad de Tarapacá. Iquique \\ 2.-Instituto Cardiológico Iquique. \\ a.-Nutricionista \\ b.-Mg. Salud Pública \\ c.-Médico cardiólogo
}

Antecedentes: La obesidad infantil es uno de los principales problemas de salud pública, y es el mejor predictor de obesidad adulta, que conlleva a futuras consecuencias, como enfermedades cardiovasculares.

Objetivo: Este estudio pretende relacionar el estado nutricional de las madres con los factores de riesgo cardiovascular (FRCV) de sus hijos con malnutrición por exceso (MNPE).

Métodos: Estudio descriptivo de corte transversal y muestreo de tipo no probabilístico por conveniencia, se efectuó en 100 diadas madres/hijos con MNPE. En cada diada se efectuaron mediciones antropométricas, peso, talla, estado nutricional según índice de masa corporal (IMC) y perímetro cintura (PC). En escolares se determinó presión arterial (PA), glicemia y consumo alimentario.

El análisis estadístico incluyó Riesgo relativo (RR) y regresión logística. Su usó el software Stata 14.
Resultados: Los promedios encontrados en los hijos fueron: edad 8.8 años, IMC/edad $21.1 \mathrm{~kg} / \mathrm{mt} 2$. En las madres, la edad promedio fue 36,9 años y el IMC $29,0 \mathrm{~kg} / \mathrm{mt} 2$. En relación con los FRCV, los escolares presentaron glicemias y presión arterial alteradas y obesidad abdominal. La malnutrición por exceso y la alteración de la percepción de las madres sobre el estado nutricional de sus hijos aumentó el RR de presentar alteración de la glicemia en 1,31 veces. La obesidad según IMC/edad presentó un RR de 1,5 y una relación positiva OR de 5,73.

Conclusiones: Existe una asociación positiva entre la MNPE de las madres y la obesidad de sus hijos. Se observó un aumento en los factores de riesgo cardiovascular de los escolares, quienes presentaban obesidad abdominal y presión arterial y glicemia alteradas. Palabras claves: Malnutrición por exceso, factores riesgo cardiovascular, obesidad.

Correspondencia:

Karina Rozas Sánchez.

Universidad de Tarapacá. Chile, Facultad de Ciencias de la Salud

Sede Iquique.

krozas78@gmail.com 


\section{Relationship between mothers' nutritional status and alterations in cardiovascular risk factors of their offsprings}

Background: Childhood obesity is one of the main public health problems. It is the best predictor of adult obesity, leading to adverse consequences, especially in relation to cardiovascular diseases.

Objective: This study aims to relate the nutritional status of mothers to the cardiovascular risk factors (CVRF) of their offsprings with excess MNPE.

Methods: A descriptive, cross-sectional study with non-probabilistic convenience sampling included 100 mother/child dyads with MNPE.

Anthropometric measurements and determination of weight, height, nutritional status (body mass index (BMI) and waist circumference (WC)), as well as blood pressure (BP), glycemia and food consumption were evaluated in schoolchildren.

The statistical analysis included Relative Risk (RR) and logistic regression, using the Stata 14 software.
Results: Average results in children included: age 8.8 years, BMI $/$ age $21.1 \mathrm{~kg} / \mathrm{mt} 2$. In mothers, mean age was 36.9 years and BMI $29.0 \mathrm{~kg} / \mathrm{mt} 2$. In relation to CVRF, schoolchildren presented altered glycemia, higher blood pressure and abdominal obesity. The MNPE and the alteration of mothers' perception of the nutritional status of their children increased the RR of altered glycemia 1.31 fold. Obesity according to $\mathrm{BMI} /$ age presented a RR of 1.5 and a positive OR 5.73 .

Conclusions: There was a positive association between the mothers' MNPE and elevated cardiovascular risk factors in school children including abdominal obesity, higher blood pressure and altered glycemia.

Keywords: Excessive malnutrition; cardiovascular risk factors, obesity. 


\section{Introducción:}

La alta prevalencia de obesidad en los niños chilenos constituye en la actualidad uno de los principales problemas de salud pública del país, alcanzando niveles semejantes a los encontrados en países desarrollados ${ }^{1}$. Debido a esto, el Ministerio de Salud de Chile, estableció como meta para la década del 2010-2020 "bajar en un 10\% la prevalencia de obesidad (de acuerdo al diagnóstico nutricional integrado) en niños menores de 6 años: de 9,6\% en 2010 a $8,6 \%$ el 2020 ".

Por otra parte, la información entregada por el Mapa Nutricional 2020 JUNAEB, publicado recientemente, en donde participó el 80,8\% de establecimientos educacionales financiados por el Estado, nos muestra que el $54,1 \%$ de los estudiantes de enseñanza regular de nuestro país presentaron MNPE.

La obesidad y el sobrepeso en la infancia pueden predisponer a desarrollar diabetes o enfermedades cardiovasculares a edades más tempranas ${ }^{2}$. El aumento de peso durante la infancia se asocia con un $25 \%$ de obesidad persistente en la edad adulta.

El desequilibrio permanente y prolongado entre la ingestión calórica y gasto energético ha sido reconocido como la principal causa de la creciente prevalencia de obesidad $^{4}$. Se han desarrollado múltiples estrategias para disminuir esta patología, pero no se ha logrado mejoría de esta situación en la población infantil.

Un metaanálisis de percepción parental de obesidad infantil indica que los padres son propensos a percibir erróneamente la proporción peso/talla de su hijo, especialmente los padres que presentan sobrepeso ${ }^{5}$.

Existen estudios que han explorado la alteración de la percepción del estado nutricional por parte de sus padres como factor de riesgo de MNPE, que demuestran que la percepción inadecuada de la proporción peso/talla en niño puede considerarse un factor de riesgo significativo para el desarrollo y/o mantenimiento del sobrepeso y obesidad, ya que $98,8 \%$ de los padres de niños con MNPE subestimaron el estado nutricional de sus hijos ${ }^{6}$.

A pesar de las múltiples estrategias para enfrentar esta patología, centradas en cambios del estilo de vida, los resultados continúan siendo desalentadores. No se ha demostrado el cambio esperado. Puede inferirse que el rol familiar, predispondría al sobrepeso y obesidad de los escolares ${ }^{7}$.

En el grupo familiar son las madres quienes ejercen un mayor control sobre la alimentación de sus hijos ${ }^{8}$. Este estudio pretende relacionar el estado nutricional de las madres con los factores de riesgo cardiovascular (FRCV) de sus hijos con malnutrición por exceso (MNPE).

\section{Métodos:}

Se trata de un estudio cuantitativo observacional, descriptivo de corte transversal desde marzo a junio del año 2019, en madres de escolares de 6 a 11 años, de primero a quinto año básico, pertenecientes a un establecimiento educacional de la ciudad de Iquique, Región de Tarapacá. El muestreo fue de tipo no probabilístico por conveniencia. Del total de la población objetivo se seleccionaron a 228 escolares, el 59\% de ellos presentaba MNPE. Finalmente, la muestra quedó conformada por 100 diadas madres/hijos MNPE.

A cada madre se le aplicó un cuestionario para establecer su edad, escolaridad, nacionalidad, etnia, número de hijos, ocupación y nivel socioeconómico. Además, se midió peso, talla, IMC y circunferencia de cintura. Se emplearon balanza digital (Seca $\left.{ }^{\circledR}\right)$, tallímetro portátil (Seca (®) y cinta métrica retráctil (Seca $\AA)$.

El diagnóstico nutricional se basó en el IMC clasificando como bajo peso (IMC <18,5), normal (IMC18,5 -24,9), sobrepeso (IMC 25- 29,9) y obesidad (IMC $\geq 30$ ).

Para evaluar la percepción del estado nutricional se utilizó un pictograma elaborado por un equipo de investigadores de la carrera de nutrición en la Universidad de Tarapacá 8 .

En escolares se midió peso, talla y perímetro de cintura utilizando los instrumentos ya señalados.

El diagnóstico nutricional se basó en el IMC promedio y su DE, clasificando como: riesgo de desnutrición (IMC/E hasta -1 DE), eutrófico (IMC/E entre - $1 \mathrm{DE}$ y +1 $\mathrm{DE})$, sobrepeso (IMC/E entre +1 DE y +2 DE), obesidad (IMC/E entre $+2 \mathrm{DE}$ y $+3 \mathrm{DE}$ ) y obesidad severa (IMC/E $>+3 \mathrm{DE})$. El perímetro de cintura se determinó de acuerdo con el sexo y edad (PC/E), clasificándolo, mediante percentiles, como normal $(<\mathrm{p} 75)$, riesgo de obesidad abdominal ( $\geq$ p75 y < p90) y obesidad abdominal ( $\geq$ p 90). Además, se midió presión arterial con monitor automático (Omron $\left.{ }^{\circledR}\right)$. So consideró como normal una glicemia $\leq \mathrm{a} 100 \mathrm{mg}-\mathrm{ml}$.

Se usaron los estadísticos apropiados según el tipo de variables fuera categórica o continua: frecuencia y porcentaje, promedio y desviación estándar, con intervalos de confianza. Se empleó análisis univariado y bivariado incluyendo OR y RR. Para análisis multivariado se usó regresión logística. Todo el análisis utilizó el programa Stata 14 y la significación estadística se fijó en p $<0.05$ 


\section{Resultados}

Características sociodemográficas: El promedio de edad de las madres fue 36,9 años (DS $\pm 7,1$ ), la escolaridad predominante fue educación técnica/profesional (36\%), la ocupación fue trabajadora (56\%), la nacionalidad fue chilena en $88 \%$ y extranjera en $12 \%$. El nivel socioeconómico (NSE) fue bajo en 53\% de las madres. En relación a los escolares su edad promedio fue 8,8 años $(\mathrm{DS} \pm 1,5)$ y $52 \%$ eran hombres (Tabla 1 ).

Indicadores antropométricos y percepción de las madres El promedio global de peso fue $72,8 \mathrm{~kg}$. (DS $\pm 14,8$ ), talla $157,7 \mathrm{~cm}$. (DS $\pm 6,9$ ), Índice de Masa Corporal (IMC) 29 $\mathrm{kg} / \mathrm{mt} 2$ (DS $\pm 5,6) 74 \%$ de las madres presentaba mal nutrición por exceso (MNPE) y riesgo cardiovascular (RCV) según circunferencia de cintura de $79 \%$.

$\mathrm{Al}$ evaluar la percepción materna sobre el estado nutricional de sus hijos encontramos que el 61,6\% de las madres de niños con MNPE subestimaron el estado nutricional, evaluándolos más delgados (Figura 1).

Indicadores antropométricos y clínicos de los escolares. El promedio de peso fue $37,7 \mathrm{~kg}$. (DS $\pm 9,6$ ), el promedio de talla 132,4 cm (DS $\pm 10,5)$, promedio IMC/Edad 21,1 $\mathrm{kg} / \mathrm{mt} 2$ (DS $\pm 2,9)$ (Tabla 1). 100\% de los escolares tenía

\begin{tabular}{|l|c|c|c|}
\hline \multirow{2}{*}{ Tabla 1. Distribución de promedios de las poblaciones estudiadas } \\
\hline HIJOS & MEDIA & DS & IC \\
\hline EDAD & 8,8 años & 1,5 & $8,5-9,1$ \\
\hline PESO & $37,7 \mathrm{~kg}$. & 9,6 & $35,8-39,6$ \\
\hline TALLA & $132,4 \mathrm{cms}$. & 10,5 & $130,2-134,4$ \\
\hline IMC/Edad & $21,1 \mathrm{~kg} / \mathrm{mt} 2$ & 2,9 & $20,5-21,6$ \\
\hline GLICEMIA & $100,01 \mathrm{mg} / \mathrm{DI}$ & 13,7 & $97,2-102,7$ \\
\hline PAS & $100,6 \mathrm{mmHg}$ & 10,7 & $98,5-102,8$ \\
\hline PAD & $69,1 \mathrm{mmHg}$ & 11,3 & $66,8-71,3$ \\
\hline & & & \\
\hline MADRES & & 7,1 & $35,5-38,3$ \\
\hline EDAD & $36,9 \mathrm{años}$ & 14,8 & $69,8-75,7$ \\
\hline PESO & $72,8 \mathrm{~kg}$. & 6,9 & $156,3-159,1$ \\
\hline TALLA & $157,7 \mathrm{cms}$. & 5,6 & $27,8-30,1$ \\
\hline IMC & 29,01 & & \\
\hline & & & \\
\hline
\end{tabular}

MNPE, $55 \%$ sobrepeso, $36 \%$ obesidad y $9 \%$ obesidad severa. El perímetro de cintura era normal en $24 \%$; en $35 \%$ indicaba riesgo de obesidad abdominal y en $41 \%$

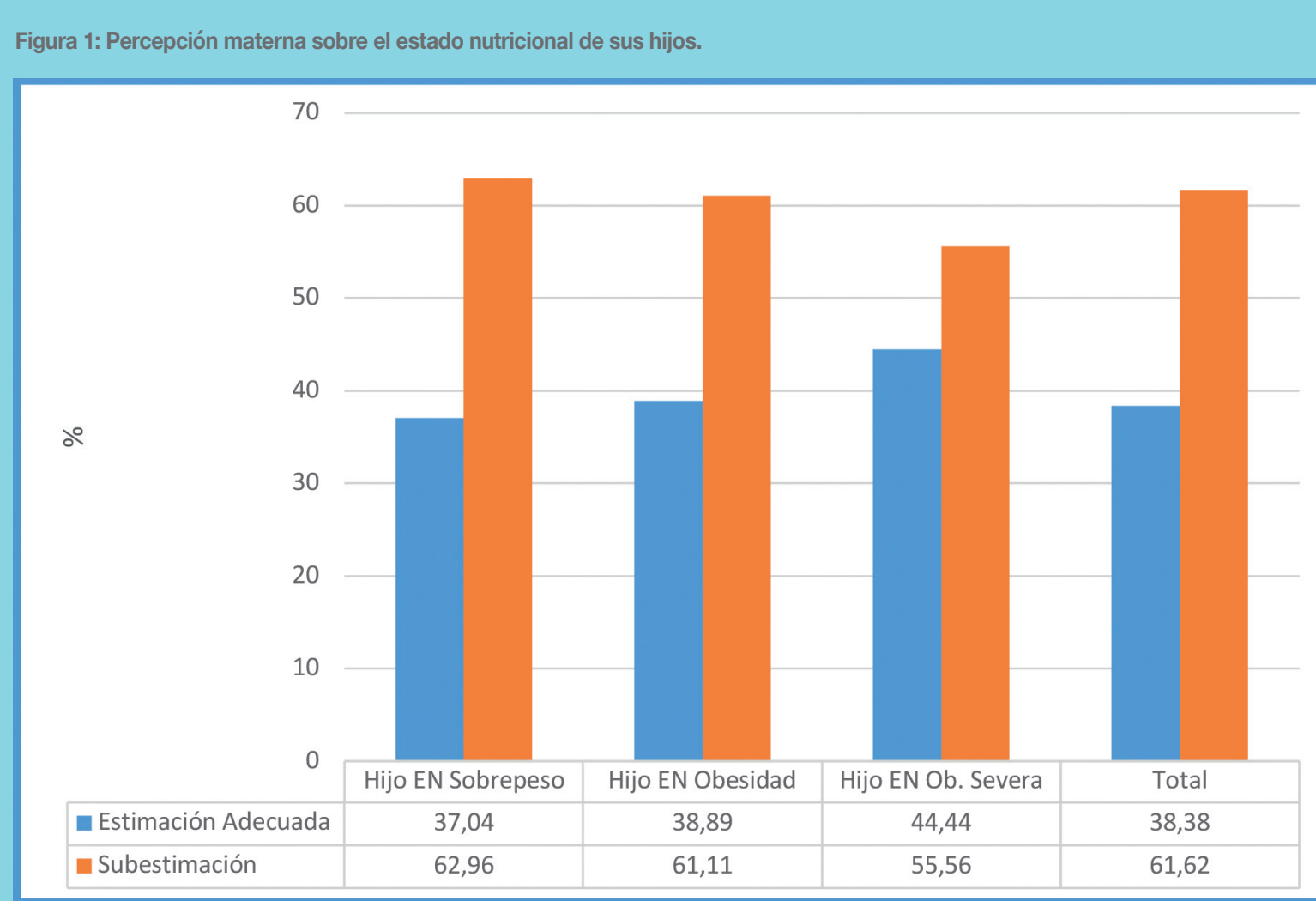


existía ya obesidad abdominal. El promedio de presión arterial sistólica fue 100,6 mmHg. (DS $\pm 10,7$ ), el de presión arterial diastólica $69,1 \mathrm{mmHg}$. (DS $\pm 11,3) ; 16 \%$ presentaba presión arterial elevada. El promedio de glicemia en ayunas fue $100 \mathrm{mg} / \mathrm{dl}(\mathrm{DS} \pm 13,7)$ y $22 \%$ tenía glicemia alterada (Tabla 1).

Consumo alimentario: La dieta era de tipo hipercalórica en $75 \%$ de los escolares, hiperproteico en $70 \%$, hiperlipídica en 77\% e hiperglucídica en 63\% (Figura 2).

Relación entre las variables del estudio con los indicadores de resultado clínico: Del total de escolares con glicemias alteradas $90,9 \%$ eran hijos de madres con MNPE; asimismo, 62,5\% de los que tenían PA alterada y $73,7 \%$ de los que presentaron obesidad abdominal, eran hijos de madres con MNPE (Figura 3). Por su parte, los hijos de madres con MNPE tienen un riesgo relativo (RR) de 1,31 (IC 1.07-1.6 p=0.04) de presentar glicemias alteradas. En hijos de madres con percepción alterada el RR de presentar obesidad era 1,5 (IC 1.14-1.98 p=0.003) (Tabla 2).

En la regresión logística se encontró una asociación positiva entre la percepción alterada de las madres, con la presencia de obesidad en sus hijos. Odds Ratio (OR) 5,73 (IC 1.92-17,1 p=0,002) (Tabla 3).

\section{Discusión:}

Los resultados presentados mostraron una relación im-

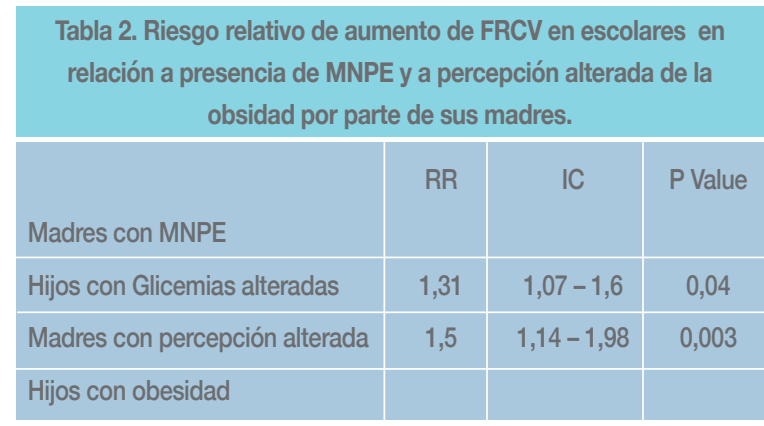

RR: riesgo relativo; IC: intervalo de confianza 95\%; MNPE: mal nutrición por exceso

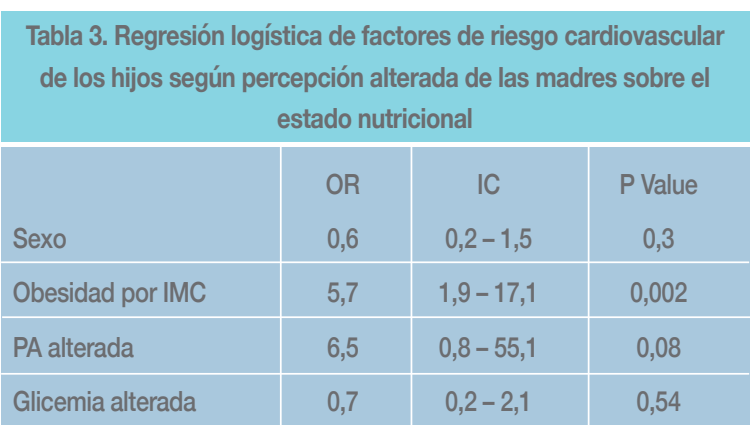

Riesgo relativo de presentar alteración de FRCV en niños, según percepción del estado nutricional por parte de sus madres.

portante entre el estado nutricional de las madres y la alteración de FRCV en sus hijos, tales como la alteración de la glicemia, elevación de la PA, obesidad abdominal y MNPE por IMC/Edad.

En nuestro país la malnutrición por exceso tanto en po-

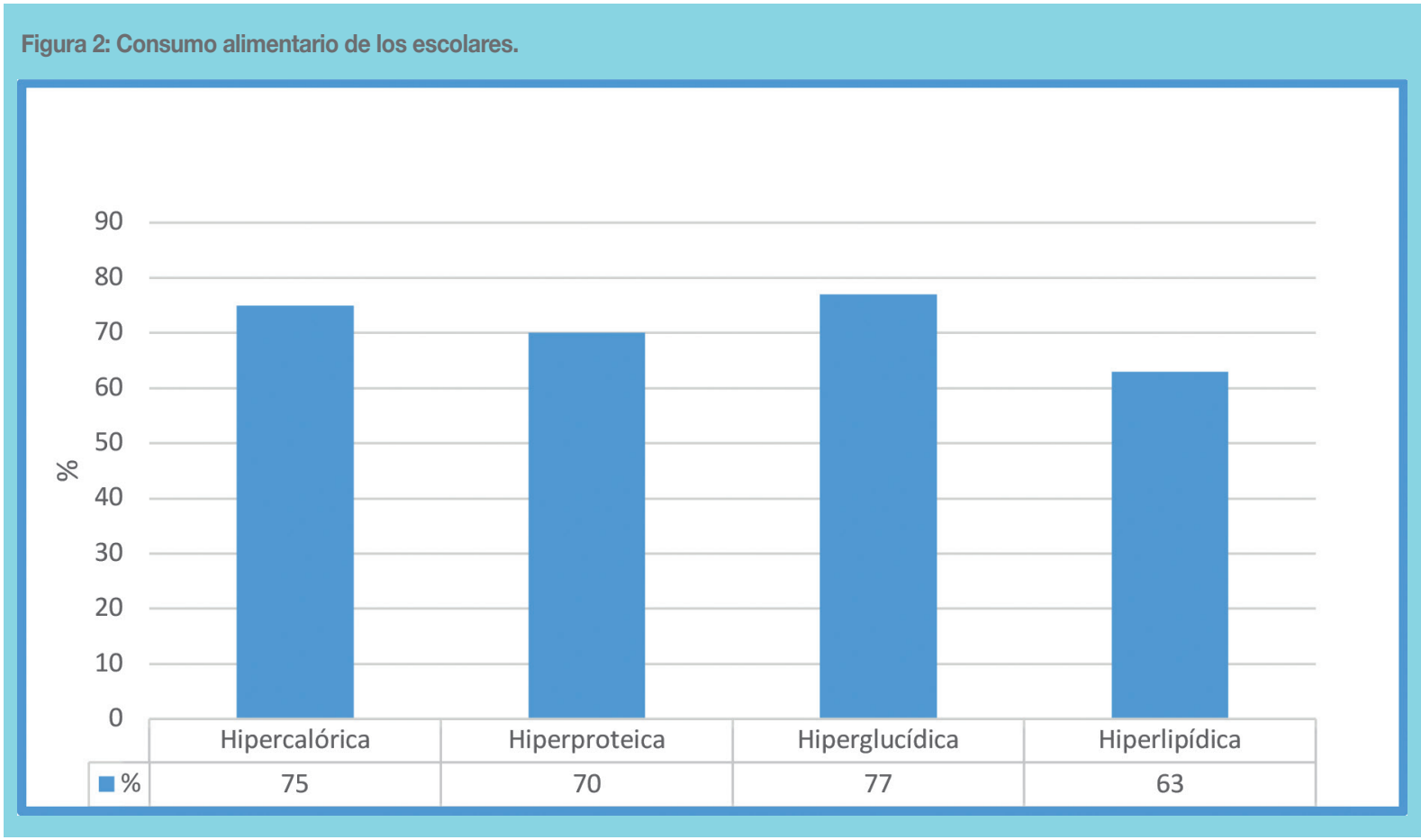




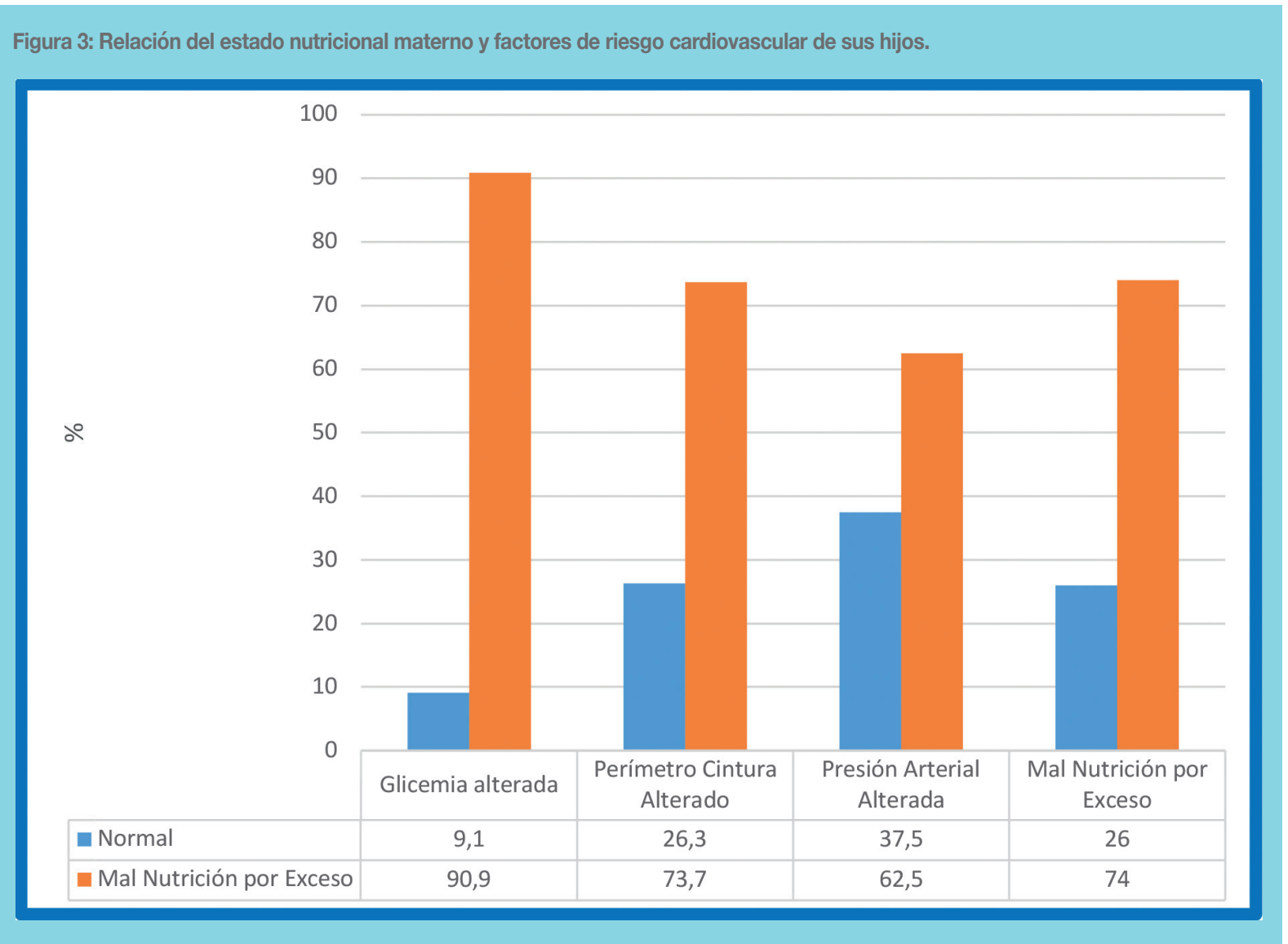

blación infantil, adolescente o adulta es un grave problema de Salud Pública. Chile es el país con mayor aumento de la obesidad infantil, alcanzando prevalencias importantes a nivel mundial ${ }^{9}$. Ello es más frecuente en familias de bajo nivel socioeconómico ${ }^{10}$. En nuestro estudio la mayoría de los escolares corresponden a un estrato socio económico bajo (53\%), hijos de madres con ocupación trabajadoras $(56 \%)$ y con un nivel bajo de educacional técnico/profesional (36\%).

Por otro lado, la información entregada por el Mapa Nutricional 2020 JUNAEB, publicado recientemente, en donde participó $80,8 \%$ de los establecimientos educacionales financiados por el estado, se observó que 54,1\% de los estudiantes de enseñanza regular presentaban MNPE. Cifras similares se encontró en el presente estudio, donde la MNPE de los escolares alcanzó un 59\%.

Estudios realizados en nuestro país han revelado que existe una subestimación de la percepción materna del estado nutricional de sus hijos. En uno de ellos se reportó que en $60 \%$ de los casos las madres tenían una percepción de un estado nutricional menos alterado que el clasificado $^{8}$. En otro estudio este porcentaje fue $54,8 \%{ }^{7}$
Por su parte, un estudio mexicano evidenció que esta distorsión fue de $98,8 \%{ }^{6}$. Nuestra investigación mostró una relación con los estudios chilenos, donde la alteración en la percepción materna alcanzó $61.6 \%$.

En relación con los FRCV de los escolares, en nuestra investigación anterior el $47 \%$ presenta obesidad abdominal según perímetro de cintura, un $35 \%$ glicemias alteradas, $31 \%$ PA alterada y un $48 \%$ de obesidad según IMC/ edad $^{8}$. En el actual estudio encontramos un $41 \%$ de obesidad abdominal, $22 \%$ y $16 \%$ de glicemias y PA alteradas respectivamente, y $45 \%$ de obesidad según IMC/edad. Estas cifras son similares en ambos estudios.

En diversos estudios se ha observado que un excesivo consumo de alimentos de alta densidad energética ricos en grasas saturadas, carbohidratos simples asociado a sedentarismo, constituyen el estilo de vida de gran parte de los niños chilenos en la actualidad ${ }^{11}$, situación que se evidencia en esta investigación, donde los escolares presentaron dietas hipercalóricas, hiperproteicas, hiperglucídicas e hiperlipídicas.

En este estudio se evidenció una relación, tanto de la MNPE como de la alteración de la percepción de las ma- 
dres con los FRCV y el estado nutricional de sus hijos, reflejando un aumento de RR de 1,31 para presentar alteración de las glicemias. La obesidad según IMC/edad presentó un RR de 1,5 y una relación positiva OR 5,73.

\section{Conclusión:}

Las madres en su mayoría tienen una alteración de la percepción sobre el estado nutricional de sus hijos, exis- tiendo además una asociación positiva entre la MNPE de las madres y la obesidad de sus hijos. De igual modo, se afectan los factores de riesgo cardiovascular de los escolares, ya que un alto porcentaje presenta obesidad abdominal, presión arterial y glicemias alteradas. La intervención nutricional debe estar dirigida principalmente en el cambio de estilos de vida del grupo familiar, enfocándose en la madre como generadora de hábitos alimentarios.

\section{Referencias:}

1.- OLIVARES S, BUSTOS N, MORENO X, LERA L, CORTEZ S. FOOD and physical activity ttitudes and practices in obese childrenand their mothers in Santiago, Chile. Rev Chil Nutr. 2006; 33(2):170-9

2.- FREEDMAN DS, KHAN LK, DIETZ WH, SRINIVASAN SR, et al. Relationship of childhood obesity to coronary heart disease risk factors in adulthood: the Bogalusa Heart Study. Pediatrics 2001;108(3):712-8.

3.- FREEDMAN DS, KATZMARZYK PT, DIETZ WH, SRINIVASAN SR, et al. Relation of body mass index and skinfold thicknesses to cardiovascular disease risk factors in children: the Bogalusa Heart Study. Am J Clin Nutr 2009; 90 (1):210-6.

4.- DECKELBAUM R, WILliAMS C. Childhood obesity: The health issue. Obes Res Clin Pract. 2001; 9(4):239-43.

5.- DOOLEN J, ALPERT PT, MILLER SK. Parental disconnect between perceived and actual weight status of children:a metasynthesis of the current research. J Am Acad Nurse Pract 2009; 21(3):160-6.

6.- KARLA L, CHÁVEZ C, RODRIGUEZ J, GUZMÁN J, SEGOVIA J, ALTAMIRANO D, et al. Alteración de la percep- ción del estado nutricional por parte de preescolares: un factor de riesgo para obesidad y sobrepeso. Arch Argent Pediatr 2016; 114(3); 237-242.

7.- NUÑO M, HEVIA M, BUSTOS C, FLORENZANO R, FRITSCH R. Distorción de la imagen corporal en madres hacia sus hijos con sobrepeso u obesidad. Rev ChilNutr Vol. 44, $\mathrm{N}^{\mathrm{a}} 1,2017$.

8.- ROZAS K, HUERTA P, PLANETT J, ARANCIBIA M, ARAYA MV. Alteración de la percepción materna sobre el estado nutricional de sus hijos ¿nuevo factor de riesgo cardiovascular?. Rev Chil Cardiol 2020; Vol.39: Número 3 216-222.

9.- KOVALSKYS I, BAY L, RAUSCH C, BERNER E. Prevalence of obesity in a population of 10- 19 year olds in a pediatric center. Rev ChilPed. 2005; 76(3): 324-325.

10.- ATALA E. Epidemiology of Obesity in Chile. Rev Med Clin-Condes. 2012; 23(2): 117-123.

11.- UAUY R, ALBALA C, KAIN J. Obesity trends in Latin America:transiting from under to overweight. J Nutr. 2001;131(3):893-9. 